\title{
APPROCHE EXPERIMENTALE DU ROLE DE RATTUS RATTUS ET DE RATTUS NORVEGICUS DANS LE FOYER DE SCHISTOSOMA MANSONI DE GUADELOUPE
}

\author{
Développement comparatif de S. mansoni \\ chez 2 hôtes naturels ( $R$. rattus et $R$. norvegicus) \\ et 2 hôtes de laboratoire (la Souris blanche et le Rat blanc)
}

D. IMBERT-ESTABLET*

RÉSUMÉ. Nous avons étudié comparativement le développement de Schistosoma mansoni chez 2 hôtes naturels, Rattus rattus et Rattus norvegicus et chez 2 hôtes de laboratoire la Souris blanche et le Rat blanc.

L'étude de la survie des Schistosomes montre que le taux de réussite de l'infestation 4 semaines après l'infestation (4 SAI) est de $31 \%$ chez $R$, rattus, de $27 \%$ chez la Souris blanche, de $14 \%$ chez le Rat blanc et de $12 \%$ chez $R$. norvegicus ; 20 semaines après l'infestation $62 \%$ des Schistosomes présents à 4 semaines sont toujours vivants chez la Souris blanche, $48 \%$ chez $R$. rattus, $8 \%$ chez $R$. norvegicus et $5 \%$ chez le Rat blanc. L'étude de la croissance montre que les Schistosomes mâles atteignent à 8 SAI une taille moyenne de $7,0 \mathrm{~mm}$ chez la Souris blanche, de $5,1 \mathrm{~mm}$ chez $R$. rattus, de $2,9 \mathrm{~mm}$ chez le Rat blanc et de $2,6 \mathrm{~mm}$ chez $R$. norvegicus. L'étude de la production d'œufs montre que $S$. mansoni produit des œufs chez $R$. rattus et $R$. norvegicus tout comme chez la Souris blanche et le Rat blanc. Cependant les œufs sont évacués par $R$. rattus et libèrent des miracidiums infestants alors que chez $R$. norvegicus les œufs produits sont stériles et ne parviennent pas dans le milieu extérieur.

Ces résultats font apparaître que la susceptibilité n'est pas toujours suivie de permissivité et qu'il existe une différence très importante entre les 2 hôtes naturels; $R$. norvegicus bien qu'acceptant $S$. mansoni ne permet pas sa transmission; $R$. rattus nettement plus favorable à la croissance et à la reproduction de $S$. mansoni autorise le passage d'œufs fertiles dans le milieu extérieur. $\boldsymbol{R}$. rattus peut done contribuer au fonctionnement du foyer de Schistosomose guadeloupéen alors que $R$. norvegicus ne doit jouer aucun rôle dans la circulation de $S$. mansoni et serait une impasse pour le parasite.

An experimental approach to the role of Rattus rattus and Rattus norvegicus in the guadelupean Schistosoma mansoni focus.

The comparative study of the development of $S$. mansoni in two natural hosts ( $R$. rattus and $R$. norvegicus) and in two laboratory hosts (the white Mouse and the white Rat).

SUMMARY. We have studied the development of Schistosoma mansoni in two natural hosts, Rattus rattus and Rattus norvegicus, and in two laboratory hosts, the white Mouse and the white Rat.

- Département de Biologie animale (Direrteur : Prof. C. Combes) Université, Avenue de Villeneuve, F 66025 Perpignan Cedex.

Accepté le 5 octobre $198 \mathrm{r}$. 
The study of survival shows that the percentage of cercariae recovered as adult worms four weeks after exposure is $31 \%$ in R. rattus, $27 \%$ in the white Mouse, $14 \%$ in the laboratory Rat and $12 \%$ in $R$. norvegicus. Twenty weeks after exposure, $62 \%$ of the Schistosomes which were present four weeks after exposure are still alive in the Mouse, $48 \%$ in $R$. rattus, $8 \%$ in $R$. norvegicus and $5 \%$ in the laboratory Rat.

The study of growth shows that the average size of males eight weeks after exposure is $7 \mathrm{~mm}$ in the Mouse, $5.1 \mathrm{~mm}$ in $R$. rattus, $2.9 \mathrm{~mm}$ in the white Rat and $2.6 \mathrm{~mm}$ in $R$. norvegicus.

The study of egg production shows that $S$. mansoni lays eggs in the four Rodents; however only mice and $R$. rattus eliminate fertile eggs containing infectious miracidia of $S$. mansoni; in $R$. norvegicus, as in the white Rat, the eggs of $S$. mansoni are not fertile and are never eliminated by the host. These results demonstrate that susceptibility is not always associated with permissivity and there is a great difference between the two natural hosts; $R$. norvegicus accepts $S$. mansoni but does not allow its transmission; $R$. rattus which is more favourable to growth and reproduction of $S$. mansoni, allows the transit and exit of fertile eggs.

These experimental data indicate that $R$. rattus is able to play a part in the dynamics of the guadeloupean schistosomiasis focus while $R$. norvegicus is unable to contribute to the circulation of $S$. mansoni and is a decoy host for the parasite.

\section{Introduction}

De nombreux Rongeurs notamment des Muridés sont susceptibles à l'infestation par Schistosoma mansoni. Dans les foyers naturels des Rongeurs sauvages ont souvent été trouvés infestés, les références étant beaucoup plus nombreuses il est vrai, pour l'Amérique que pour l'Afrique. Cette réceptivité des Rongeurs sauvages ne peut en faire de véritables réservoirs de la maladie que si elle est accompagnée d'un développement complet des Schistosomes avec évacuation d'œufs viables libérant des miracidiums infestants.

Des travaux récents dans le foyer de Schistosomose de Guadeloupe (Combes et Delattre, 1981) ont révélé une forte prévalence de $S$. mansoni chez Rattus rattus et Rattus norvegicus. Nous avons réalisé une étude expérimentale du développement et de la reproduction de $S$. mansoni chez ces deux hôtes naturels et chez deux hôtes de laboratoire, la Souris blanche et le Rat blanc, la première classiquement reconnue comme un hôte favorable, le second comme un hôte défavorable. Par cette étude nous envisageons d'évaluer comparativement le degré d'adaptation de $S$. mansoni à ces différents hôtes et de situer la "valeur hôte" de $R$. rattus et des $R$. norvegicus, très peu étudiée jusqu'ici, par rapport à celle de la Souris blanche et du Rat blanc.

\section{Matériel et techniques}

\section{Matériel}

La souche de $S$. mansoni utilisée provient de $R$. rattus infestés naturellement et capturés en Guadeloupe. Pour les expériences concernant les Rats cette souche a été entretenue sur $R$. rattus (1 ou 2 passages); pour les expériences concernant les Souris cette même souche a été entretenue sur Souris (1, 2 ou 3 passages successifs). 
La souche de Biomphalaria glabrata utilisée est originaire de Guadeloupe.

Les Rats sauvages $R$. rattus et $R$. norvegicus sont d'ascendance guadeloupéenne. Les Souris blanches sont de souche Swiss $\mathrm{OF}_{1}$.

Les Rats blancs sont de souche Wistar.

\section{Techniques}

\section{INFESTATIONS}

Les Rongeurs sont infestés sous anesthésie générale par pénétration transabdominale des cercaires (Smithers et Terry, 1965 ; Erickson, 1974). 1000 cercaires sont utilisées pour l'infestation de chaque Rat, 150 pour l'infestation de chaque Sour le temps d'exposition étant de 30 minutes.

\section{RÉCUPÉRATION DES GUFS}

La récupération des œufs contenus dans les fèces se fait après homogénéisation dans une solution de $\mathrm{C} 1 \mathrm{Na}$ à $9 \%$ des fèces de 24 heures, puis filtration sur une colonne de filtres en acier $(500 \mu, 200 \mu, 160 \mu)$. Le filtrat est ensuite décanté et plusieurs lavages successifs sont effectués; le volume est amené à $300 \mathrm{cc}$ et les œufs sont dénombrés par comptage direct dans dix prélèvement de 1cc.

La récupération des œufs contenus dans le foie, les poumons et l'intestin est réalisée par la technique de digestion à la potasse de Cheever (1968).

\section{RÉCUPÉRATION DES SCHISTOSOMES}

La récupération des Schistosomes localisés dans le système porte mésentérique se fait par perfusion selon la technique de Duwall et Dewitt (1967). De nombreux adultes se trouvant dans le foie et les poumons des Rats, la perfusion hépatique est complétée par une dilacération de ces organes permettant la récupération des parasites.

\section{Mensuration DES SCHISTOSOMES}

Les mensurations des Schistosomes sont effectuées après anesthésie au Nembutal à $0,08 \%$.

\section{Résultats}

\section{Survie}

Des recensements de la population de $S$. mansoni ont été effectués à divers intervalles de temps après l'infestation des Rongeurs (tableaux $I$ à $I V$ ).

A partir de ces données nous avons construit les courbes de survie et les histogrammes (fig. 1 et 2).

Ces résultats montrent que :

- à 4 SAI (4 semaines après infestation) le nombre de parasites récupérés par rapport au nombre de cercaires utilisées pour l'infestation (c'est-à-dire le taux de 
réussite de l'infestation à $4 \mathrm{SAI}$ ) est nettement plus élevé chez la Souris blanche et $R$. rattus $(27 \%$ et $31 \%$ ) que chez le Rat Wistar et $R$. norvegicus ( $14 \%$ et $12 \%$ ) ; - entre 4 et 20 SAI la population décroît beaucoup plus fortement chez le Rat Wistar et $R$. norvegicus que chez la Souris blanche et $R$. rattus;

- à $52 \mathrm{SAI}$, soit une année après l'infestation, des Schistosomes sont encore présents chez $R$. rattus.

Tableau I. - Localisations et effectifs de $S$. mansoni chez le Rat blanc (Wistar) à divers laps de temps après l'infestation (1 000 cercaires sont utilisées pour l'infestation de chaque Rat).

\begin{tabular}{|c|c|c|c|c|c|}
\hline \multirow{2}{*}{$\begin{array}{l}\text { No d'ordre } \\
\text { des Rats } \\
\text { perfusés }\end{array}$} & \multirow{2}{*}{$\begin{array}{c}\text { Nombre } \\
\text { de }\end{array}$} & \multicolumn{4}{|c|}{ Nombre de Schistosomes } \\
\hline & & $\begin{array}{l}\text { Système } \\
\text { porte-mésentérique }\end{array}$ & Foie & Poumons & Total \\
\hline 1 & 4 & 131 & 3 & 0 & 134 \\
\hline 2 & 4 & 184 & 1 & 0 & 185 \\
\hline 3 & 4 & 101 & 0 & 0 & 101 \\
\hline 4 & 6 & 23 & 66 & 0 & 89 \\
\hline 5 & 6 & 64 & 30 & 0 & 94 \\
\hline 6 & 6 & 29 & 33 & 0 & 62 \\
\hline 7 & 12 & 3 & 26 & 0 & 29 \\
\hline 8 & 12 & 40 & 21 & 0 & 61 \\
\hline $\begin{array}{l}\circ \\
9\end{array}$ & 12 & 42 & 16 & 0 & 58 \\
\hline 10 & 12 & 30 & 57 & 0 & 87 \\
\hline 11 & 16 & 29 & 4 & 0 & 33 \\
\hline 12 & 20 & 2 & 9 & 0 & 11 \\
\hline 13 & 20 & 4 & 0 & 0 & 4 \\
\hline
\end{tabular}

TABLEAU II. - Localisations et effectifs de $S$. mansoni chez $R$. norvegicus à divers laps de temps après l'infestation (1000 cercaires sont utilisées pour l'infestation de chaque Rat).

\begin{tabular}{|c|c|c|c|c|c|}
\hline \multirow{2}{*}{$\begin{array}{l}\text { No d'ordre } \\
\text { des Rats } \\
\text { perfusés }\end{array}$} & \multirow{2}{*}{$\begin{array}{c}\text { Nombre } \\
\text { de } \\
\text { SAI }\end{array}$} & \multicolumn{4}{|c|}{ Nombre de Schistosomes } \\
\hline & & $\begin{array}{l}\text { Système } \\
\text { porte-mésentérique }\end{array}$ & Foie & Poumons & Total \\
\hline 1 & 4 & 153 & 1 & 0 & 154 \\
\hline 2 & 4 & 88 & 3 & 0 & 91 \\
\hline 3 & 6 & 75 & 42 & 1 & 118 \\
\hline 4 & 6 & 25 & 75 & 0 & 100 \\
\hline 5 & 6 & 29 & 8 & 0 & 37 \\
\hline 6 & 9 & 89 & 4 & 0 & 89 \\
\hline 7 & 12 & 55 & 11 & 4 & 70 \\
\hline 8 & 12 & 54 & 12 & 3 & 69 \\
\hline 9 & 16 & 2 & 7 & 0 & 9 \\
\hline 10 & 18 & 10 & 8 & 0 & 18 \\
\hline 11 & 20 & 4 & 8 & 0 & 12 \\
\hline 12 & 20 & 5 & 4 & 0 & 9 \\
\hline
\end{tabular}




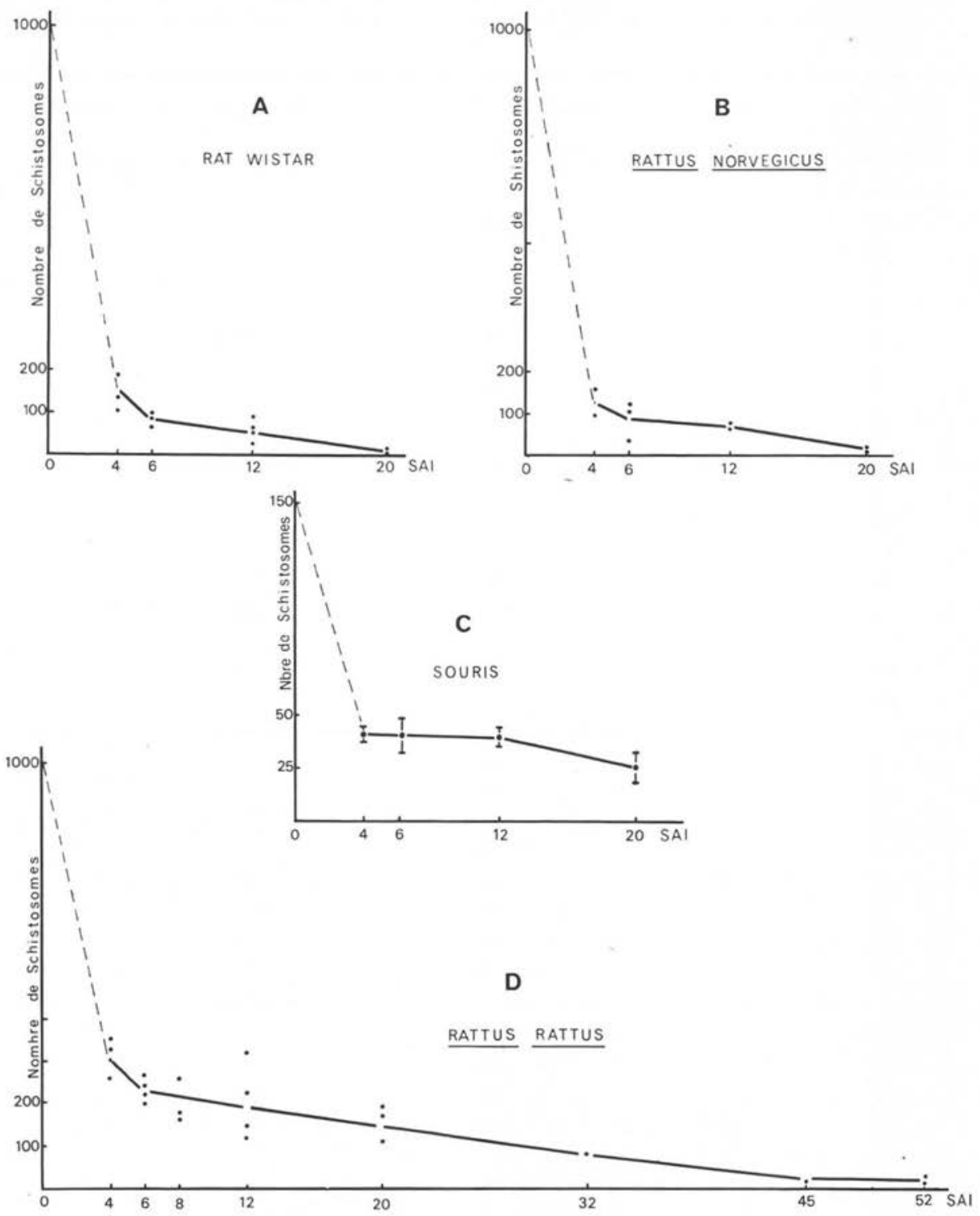

FIG. I. - Courbes de survie de S. mansoni chez deux hôtes de laboratoire (A, C) et deux hôtes naturels $(\mathrm{B}, \mathrm{D})$. 
TABLEAU III. - Localisations et effectifs de $S$. mansoni chez la Souris à divers laps de temps après l'infestation (150 cercaires sont utilisées pour l'infestation de chaque Souris).

\begin{tabular}{|c|c|c|c|}
\hline $\begin{array}{l}\text { No d'ordre } \\
\text { des Souris } \\
\text { perfusées }\end{array}$ & $\begin{array}{c}\text { Nombre } \\
\text { de } \\
\text { SAI }\end{array}$ & $\begin{array}{c}\text { Nombre de Schistosomes } \\
\text { Système } \\
\text { porte-mésentérique }\end{array}$ & $\begin{array}{l}\text { Moyenne avec intervalle } \\
\text { de confiance } \\
\text { (Coef. de sécurité } 95 \% \text { ) }\end{array}$ \\
\hline 1 & \multirow{8}{*}{4} & 39 & \multirow{8}{*}{$40,50 \pm 2,65$} \\
\hline 2 & & 37 & \\
\hline 3 & & 42 & \\
\hline 4 & & 43 & \\
\hline 5 & & 42 & \\
\hline 6 & & 5. 44 & \\
\hline 7 & & 35 & \\
\hline 8 & & 42 & \\
\hline 9 & \multirow{8}{*}{6} & 38 & \multirow{8}{*}{$40,25 \pm 8,28$} \\
\hline 10 & & 39 & \\
\hline 11 & & 19 & \\
\hline 12 & & 42 & \\
\hline 13 & & 42 & \\
\hline 14 & & 44 & \\
\hline 15 & & 44 & \\
\hline 16 & & 54 & \\
\hline 17 & \multirow{8}{*}{12} & 50 & \multirow{8}{*}{$40 \pm 4,44$} \\
\hline 18 & & 43 & \\
\hline 19 & & 35 & \\
\hline 20 & & 34 & \\
\hline 21 & & 39 & \\
\hline 22 & & 43 & \\
\hline 23 & & 40 & \\
\hline 24 & & 36 & \\
\hline 25 & \multirow{8}{*}{20} & 16 & \multirow{8}{*}{$25,12 \pm 7,20$} \\
\hline 26 & & 35 & \\
\hline 27 & & 22 & \\
\hline 28 & & 30 & \\
\hline 29 & & 17 & \\
\hline 30 & & 31 & \\
\hline 31 & & 15 & \\
\hline 32 & & 35 & \\
\hline
\end{tabular}



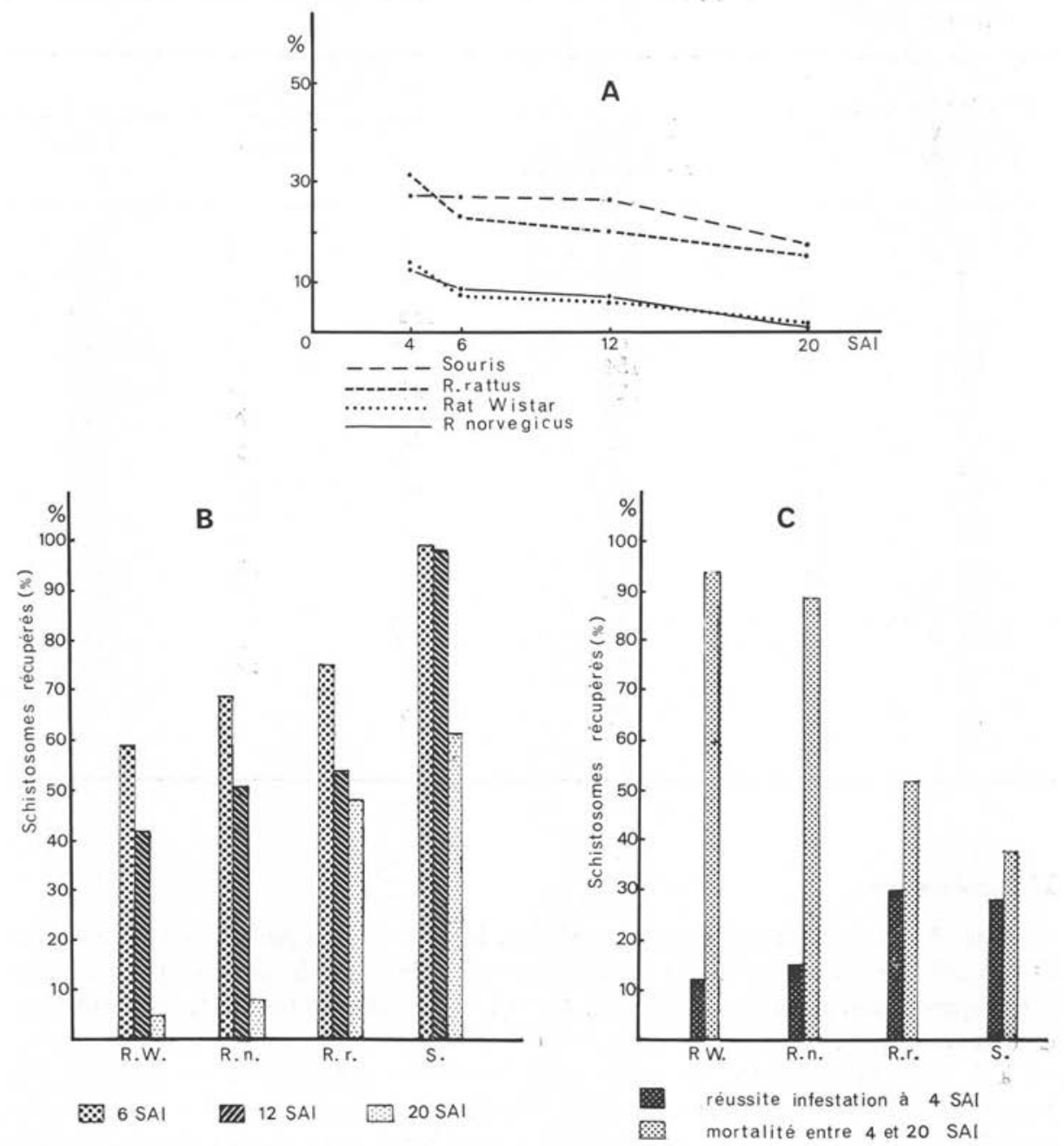

FIG. 2. - A. Population totale de Schistosomes présents à 4, 6, I 2 et 20 SAI, exprimée en pourcentage de la population cercarienne utilisée pour l'infestation.

B. Taux de survie des Schistosomes entre 4 et 20 SAI. (R.W. = Rat Wistar ; R.n. $=$ Rattus norvegicus; R.r. = Rattus rattus ; S. = Souris).

C. Comparaison entre le taux de réussite de l'infestation à 4 SAI et le taux de mortalité entre 4 et 20 SAI. 
TABLEAU IV. - Localisations et effectifs de $S$. mansoni chez $R$. rattus à divers laps de temps après l'infestation (1 000 cercaires sont utilisées pour l'infestation de chaque Rat).

\begin{tabular}{|c|c|c|c|c|c|}
\hline \multirow{2}{*}{$\begin{array}{l}\text { No d'ordre } \\
\text { des Rats } \\
\text { perfusés }\end{array}$} & \multirow{2}{*}{$\begin{array}{c}\text { Nombre } \\
\text { de } \\
\text { SAI }\end{array}$} & \multicolumn{4}{|c|}{ Nombre de Schistosomes } \\
\hline & & $\begin{array}{c}\text { Système } \\
\text { porte-mésentérique }\end{array}$ & Foie & Poumons & Total \\
\hline 1 & 4 & 312 & 14 & 0 & 326 \\
\hline 2 & 4 & 349 & 9 & 0 & 358 \\
\hline 3 & 4 & 253 & 7 & 0 & 260 \\
\hline 4 & 6 & 60 & 118 & 64 & 242 \\
\hline 5 & 6 & 164 & 98 & 6 & 268 \\
\hline 6 & 6 & 154 & 8 & 34 & 196 \\
\hline 7 & 6 & 125 & 51 & 44 & 220 \\
\hline 8 & 8 & 32 & 40 & 136 & 172 \\
\hline 9 & 8 & 23 & 10 & 121 & 154 \\
\hline 10 & 8 & 2 & 2 & 154 & 258 \\
\hline 11 & 12 & $14 \overline{5}$ & 38 & 139 & 322 \\
\hline $\begin{array}{l}11 \\
12\end{array}$ & 12 & 26 & 5 & 114 & 145 \\
\hline 13 & 12 & 57 & 10 & 51 & 118 \\
\hline 14 & 12 & 52 & 44 & 128 & 224 \\
\hline 15 & 20 & 10 & 4 & 150 & 164 \\
\hline 16 & 20 & 10 & 4 & 172 & 186 \\
\hline 17 & 20 & 47 & 34 & 23 & 104 \\
\hline 18 & 32 & 14 & 5 & 120 & 139 \\
\hline 19 & 45 & 8 & 5 & 0 & 13 \\
\hline 20 & 52 & 9 & 2 & 0 & 11 \\
\hline 21 & 52 & 22 & 2 & 4 & 28 \\
\hline
\end{tabular}

\section{Croissance}

Des Schistosomes mâles récupérés chez les divers Rongeurs à 4 et 8 SAI ont été anesthésiés et mesurés. Les distributions de fréquence de taille et les moyennes sont représentées graphiquement ( $f i g .3$ et 4). Les résultats obtenus nous permettent de constater que :

- à 4 SAI les différences de taille entre les divers échantillons ne sont pas très importantes, mais on observe un léger retard de croissance pour les Schistosomes recueillis chez $R$. norvegicus et une légère avance pour ceux s'étant développés chez la Souris;

- à 8 SAI des écarts plus importants apparaissent; c'est la Souris blanche qui permet la meilleure croissance des Schistosomes mâles, suivie d'assez près par $R$. rattus. Le Rat Wistar et $R$. norvegicus par contre, paraissent ne pas offrir l'environnement convenable au développement de $S$. mansoni.

Cette étude comparative de la croissance de $S$. mansoni chez 4 Rongeurs différents confirme un fait que Cioli et al. (1977) ont signalé, à savoir que les influences de l'hôte sur la croissance du parasite sont beaucoup plus marquées après la $4^{\mathrm{e}}$ semaine qui suit l'infestation. Ces auteurs, après transplantation de Schistosomes adultes 

4 SAI
8 SAI
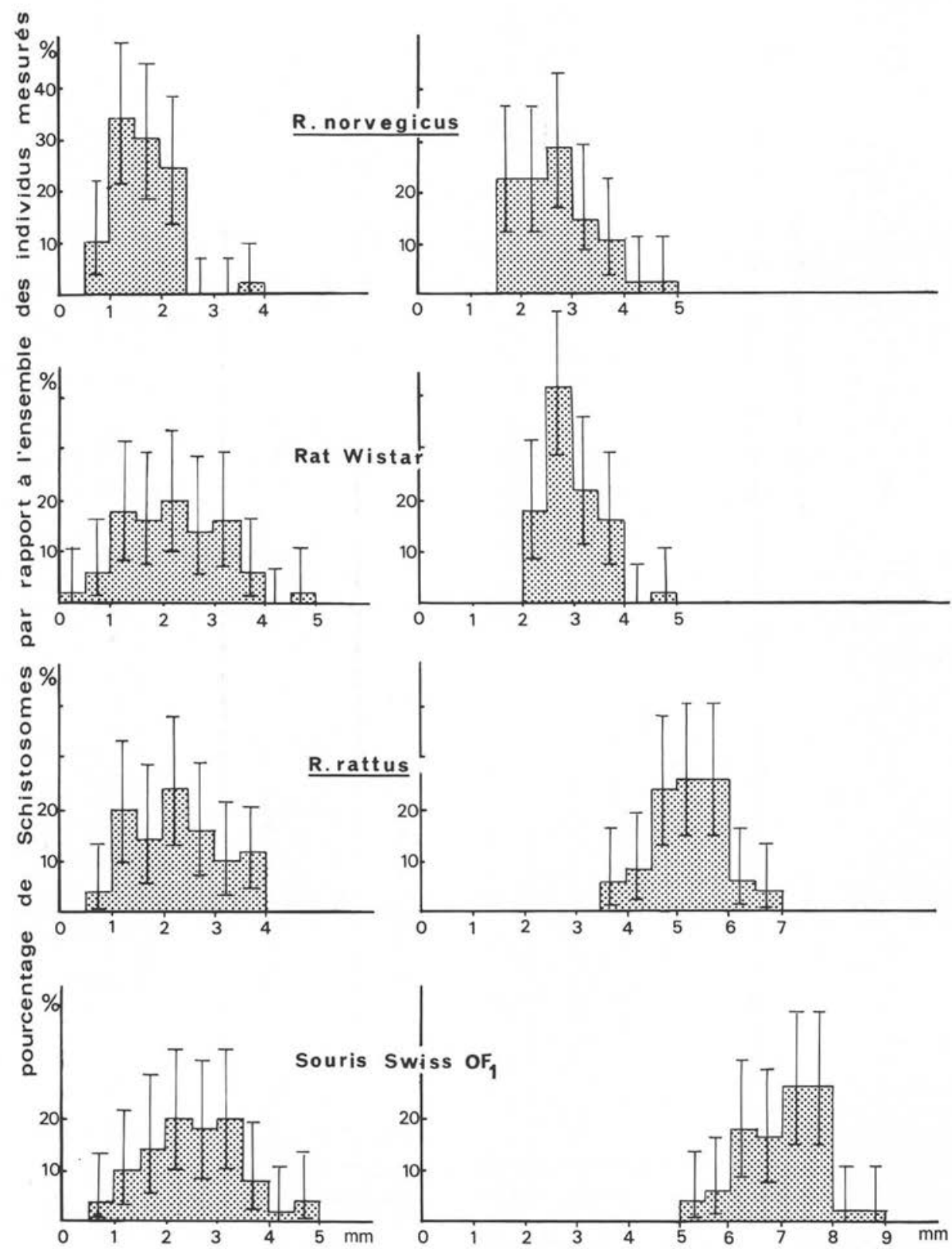

FIG. 3. - Distribution des fréquences de taille des Schistosomes mâles récupérés à 4 et 8 SAI (intervalles de confiance au coefficient de sécurité $95 \%$ ). 


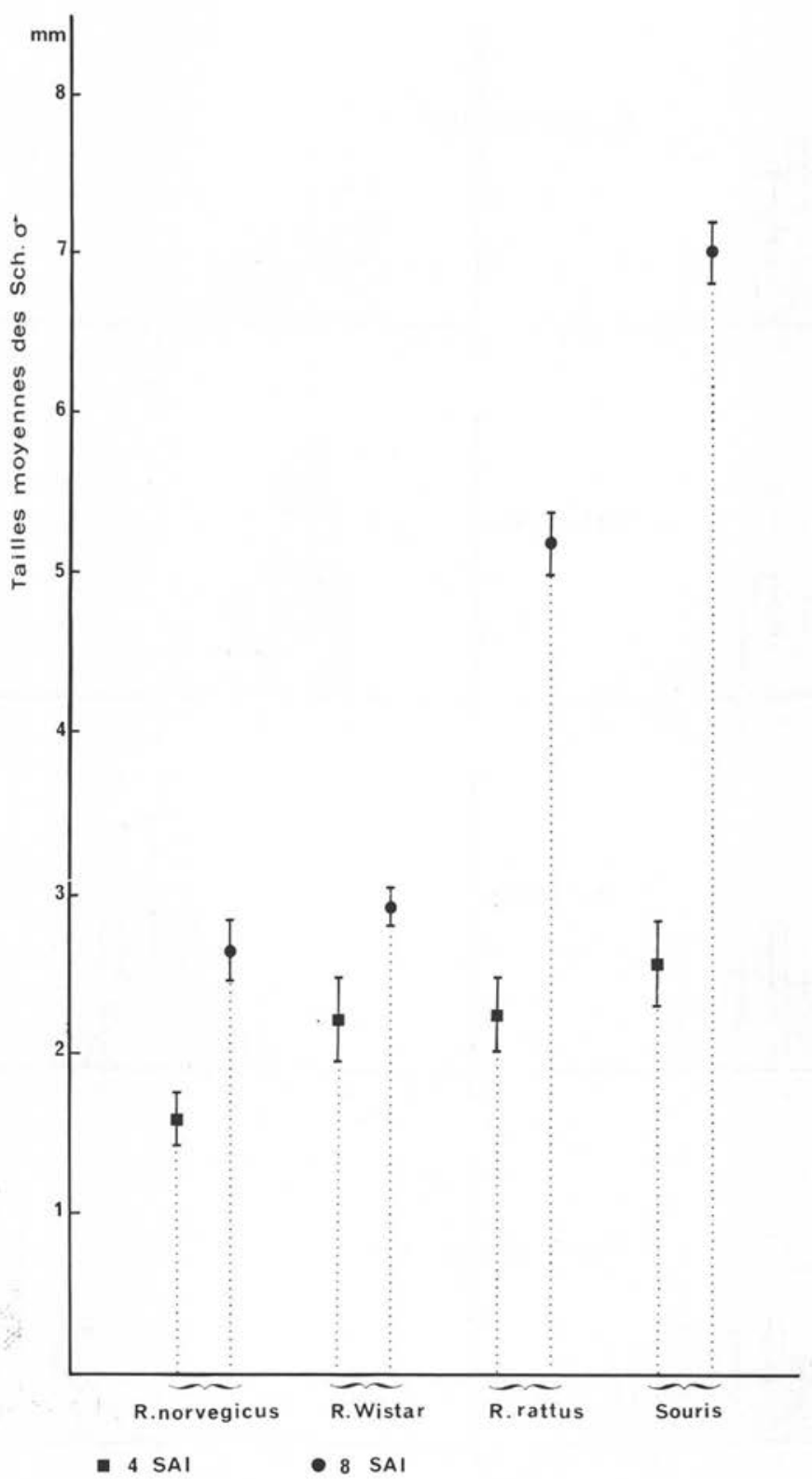

Fig. 4. - Valeur moyenne (avec intervalle de confiance au coefficient de sécurité de $95 \%$ ) des tailles des Schistosomes mâles prélevés chez 4 Rongeurs à 4 et 8 SAI. 
d'un hôte défavorable (Rat blanc) à un hôte favorable (Hamster, Souris blanche) ont démontré que l'arrêt de la croissance n'est pas un phénomène irréversible; ces variations de taille des adultes d'une même espèce selon l'hôte où ils se trouvent doivent donc être considérées comme une expression de la compatibilité du système hôte-parasite.

\section{Production d'œufs}

Les résultats concernant l'évaluation du nombre d'œufs contenus dans les fèces au cours de la ge SAI figurent dans le tableau $V$.

Les résultats concernant la charge en œufs des divers organes (foie, poumons, intestin) au terme de la 9 e SAI figurent dans le tableau VI.

TABleau V. - Nombre d'œufs contenus dans les fèces des 4 Rongeurs au cours de la $9^{\mathrm{e}}$ semaine après l'infestation.

\begin{tabular}{lccc}
\hline \multicolumn{1}{c}{ Féces } & $\begin{array}{l}\text { Nombre d'œufs } \\
\text { pendant 7 jours } \\
\text { (9e SAI) }\end{array}$ & $\begin{array}{c}\text { Nombre de miracidiums } \\
\text { (après 5 h de mise en } \\
\text { conditions favorables) }\end{array}$ & $\begin{array}{c}\text { Nombre de couples } \\
\text { à localisation } \\
\text { porte-mésentérique }\end{array}$ \\
\hline Rattus norvegicus & 0 & 0 & 11 \\
Rat Wistar & 0 & 0 & 42 \\
Rattus rattus & 725 & 22 & 53 \\
Souris & 7000 & 725 & 19 \\
\hline
\end{tabular}

TABleAU VI. - Charge en œufs de divers organes au terme de la $9^{\mathrm{e}}$ semaine après l'infestation des 4 Rongeurs.

\begin{tabular}{|c|c|c|c|c|c|}
\hline \multirow[b]{2}{*}{$9 \mathrm{SAI}$} & \multicolumn{3}{|c|}{ Nombre d'œufs } & \multicolumn{2}{|c|}{ Nombre de couples } \\
\hline & $\begin{array}{c}\text { Paroi } \\
\text { intestinale } \\
\text { œufs } / \mathrm{cm}\end{array}$ & $\begin{array}{c}\text { Foie } \\
œ u f s / g r\end{array}$ & $\begin{array}{c}\text { Poumons } \\
œ u f s / g r\end{array}$ & Pulmonaires & $\begin{array}{l}\text { Hépato- } \\
\text { mésentériques }\end{array}$ \\
\hline Rattus norvegicus & 0 & 333 & 33 & 0 & 11 \\
\hline Rat Wistar & 0 & 1348 & 0 & 0 & 36 \\
\hline Rattus rattus & 43 & 3787 & 1493 & 9 & 41 \\
\hline Souris & 200 & 3004 & 0 & 0 & 19 \\
\hline
\end{tabular}

Ces résultats montrent que :

- chez le Rat Wistar et chez $R$. norvegicus aucun œuf de $S$. mansoni ne parvient dans le milieu extérieur ; on constate chez ces deux Rongeurs l'absence totale d'œufs dans la paroi intestinale et bien qu'un grand nombre d'œufs aient été dénombrés dans le foie aucun n'a permis l'obtention de miracidium; 
- chez la Souris blanche et chez $R$. rattus le passage des œufs de $S$. mansoni dans le milieu extérieur se produit ; ces œufs libèrent des miracidiums viables (nous avons vérifié que ces miracidiums sont infestants avec des taux de réussite semblables dans les deux cas). La charge en œufs du foie est importante et du même ordre chez les deux espèces ; le nombre d'œufs présents dans la paroi intestinale est plus élevé chez la Souris que chez $R$. rattus ce qui semble aller de pair avec le plus grand nombre d'œufs retrouvés dans les fèces. Si l'on ramène l'évacuation journalière d'œufs au nombre de couples de Schistosomes porte-mésentériques on constate que $R$. rattus reste très en deçà de la Souris blanche (1 œuf pour 24 heures et par couple contre 54). Des œufs sont présents en grand nombre dans les poumons de $R$. rattus, en très petit nombre chez $R$. norvegicus et sont absents chez le Rat Wistar et la Souris blanche.

De nombreux travaux ont été effectués sur la production et le passage des œufs de $S$. mansoni chez divers hôtes Vertébrés notamment la Souris blanche (Moore et Sandground, 1956; Kuntz, 1961 ; Koura, 1970; FAN, 1974) et le Rat blanc (Koppish, 1937 ; Ritchie et al., 1963) ; les résultats (chez la Souris blanche) de Moore et Sandground (66 œufs/24 heures/femelle) et de Kuntz (60œufs/24 heures/femelle) sont très proches de ceux que nous avons obtenus chez le même hôte.

Tous les travaux effectués chez le Rat blanc montrent qu'il n'y a pas évacuation d'œufs dans les fèces, et que les œufs retenus dans le foie ne libèrent qu'exceptionnellement des miracidiums viables mais par ailleurs non infestants (Ritchie et al., 1963). Nos résultats sont en accord avec ces données.

La souche de S. mansoni guadeloupéenne que nous avons utilisée se comporte donc de manière totalement " classique " vis-à-vis des hôtes de laboratoire, Souris blanche et Rat Wistar. Il nous paraît donc important de souligner les résultats positifs obtenus pour $R$. rattus tant en ce qui concerne l'évacuation d'œufs que la viabilité et l'infectivité de ces œufs. Il n'est pas étonnant par ailleurs de noter que les résultats obtenus avec les $R$. norvegicus sauvages sont pratiquement identiques à ceux obtenus habituellement avec les Rats blancs de laboratoire.

\section{Conclusion}

Cette étude comparative nous permet d'établir un " classement » des Rongeurs basé sur leur qualité d'hôte pour S. mansoni et de situer les hôtes naturels rencontrés en Guadeloupe par rapport à la Souris blanche et au Rat blanc de laboratoire (fig. 5). $R$. norvegicus, tout comme le Rat Wistar, autorise la pénétration de $S$. mansoni mais la mortalité des adultes y est très élevée, les survivants n'ont qu'une croissance incomplète et une fécondité réduite, les œufs ne contiennent pas de miracidiums viables et ne parviennent pas dans le milieu extérieur; ces deux Rougeurs sont des hôtes non permissifs. Il est vraisemblable que les $R$. norvegicus sauvages présentent des mécanismes de défense semblables à ceux qui ont été démontrés chez leur descendant de laboratoire. Bien que pouvant être infestés dans les foyers des Caraïbes et d'Amérique du Sud, $R$. norvegicus n'est probablement pas un réservoir de la 


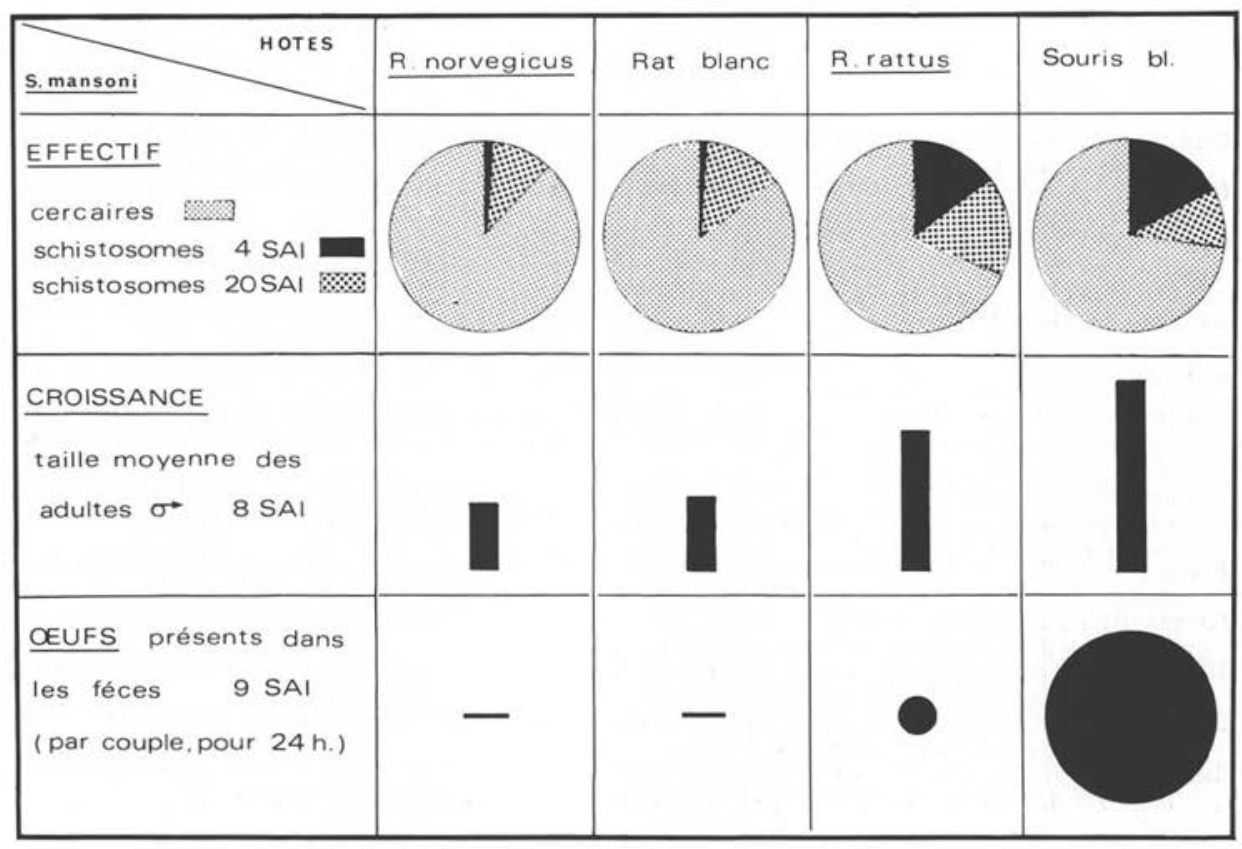

FIG. 5. - Influence du Rongeur sur le développement de S. mansoni.

maladie. Au contraire $R$. rattus qui offre un environnement presque aussi favorable à $S$. mansoni que la Souris blanche et qui permet le passage à l'extérieur d'œufs capables d'assurer la poursuite du cycle parasitaire peut parfaitement avoir une part de responsabilité dans l'entretien de certains foyers. $R$. rattus et $R$. norvegicus, a priori tous deux susceptibles d'être de véritables réservoirs de la Schistosomose en Guadeloupe, auraient ainsi des rôles différents et même opposés dans les sites de transmission. Cependant, nous ne devons pas perdre de vue que dans nos expériences l'infestation de chaque Rongeur a été faite par une exposition unique aux cercaires alors que dans le milieu naturel existe la possibilité d'infestations successives. Il est donc nécessaire de compléter les données acquises au cours de ce travail par une analyse de la parasitose chez des Rongeurs infestés dans le milieu naturel.

Remerciements : Nous remercions vivement le Docteur M.-Cl. Durette-Desset du Muséum National d'Histoire Naturelle de Paris, qui a mis au point un élevage de Rats Sauvages originaires de Guadeloupe et qui nous a fourni les $R$. rattus et $R$. norvegicus utilisés au cours de cette étude.

Ce travail a reçu le support financier du programme spécial PNUD, Banque Mondiale, O.M.S. de Recherche et de Formation concernant les maladies tropicales. 


\section{BIBLIOGRAPHIE}

Chenver A. W. : Conditions affecting the accuracy of potassium hydroxyde digestion techniques for counting Schistosoma mansoni eggs in tissues. Bull. Wld. Hlth. Org., 1968, 39, 328-331.

Cioli D., KNopf P. M., SEnfr W. : A study of Schistosoma mansoni transferred into permissive and non permissive hosts. Internation. J. Parasit., 1977, 7, 293-297.

Combes C., Delatrre P. : Principaux paramètres de l'infestation des Rats (Rattus rattus et Rattus norvegicus) par Schistosoma mansoni dans un foyer de Schistosomose intestinale de la région caraïbe. Oecol. Applic., 1981, 2, 63-79.

Duwall R. H., DewitT W. B. : An improved perfusion technique for recovering adult Schistosomes from laboratory animals. Am. J. Trop. Med. Hyg., I967, 16, 483-486.

ERICKSON D. G. : An efficient technique for exposure of Rodents to Schistosoma haematobium. J. Parasitol., 1974, 60, 553-554.

FAN PING-CHIN : Quantitative comparison of the production development and distribution of eggs of Schistosoma mansoni in mice infected with one and multi-paired worms. Yonsei Rep. trop. Med., I974, S, II7-129.

KoppISH E. : Studies on schistosomiasis mansoni in Puerto Rico. IV. The pathological anatomy of experimental schistosomiasis mansoni in Rabbit and Albino Rat. J. Pub. Hith. trop. Med., 1937, I3, I-54.

KoURA M. : The relation between egg production and worm burden in experimental schistosomiasis. I. Worm burden. J. Egypt. Med. Ass., 1970, 53, 598-602.

KunTz R. E. : Passage of eggs by hosts infected with Schistosoma mansoni with emphasis on Rodents. J. Parasitol., 1961, 47, 905-909.

MOORE D. V., SANDGROUND J. H. : The relative egg producing capacity of Schistosoma mansoni and Schistosoma japonicum. Am. J. trop. Med. Hyg., 1956, 5, 831-840.

Ritchie L. S., GARSON S., KNIGHT W. : The biology of Schistosoma mansoni in laboratory Rats. J. Parasitol., 1963, 49, 571-577.

Smithers S. R., TERRY R. J. : The infection of laboratory hosts with cercariae of Schistosoma mansoni and the recovery of the adult worms. Parasitol., 1965, 55, 695-700. 